\title{
Measuring user preferences in virtual reality (VR): 2D versus 3D urban geovisualizations of topographic data
}

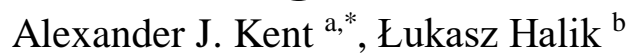 \\ ${ }^{a}$ School of Human and Life Sciences, Canterbury Christ Church University, United Kingdom, alexander.kent@canterbury.ac.uk \\ ${ }^{b}$ Department of Cartography and Geomatics, Adam Mickiewicz University in Poznań, Poland, lukasz.halik@amu.edu.pl \\ * Corresponding author
}

Keywords: Virtual Reality, VR, 3D, 2D, urban area, topographic data

\begin{abstract}
:
Virtual reality (VR) is a display and control technology that provides an interactive computer-generated threedimensional environment to a user, often via a Head Mounted Display (HMD). VR delivers an immediate and immersive sensory experience of simulated worlds (which may or may not resemble reality), particularly of environments that might otherwise be physically inaccessible to the user due to their location, scale, time or danger. Although the first VR systems began to emerge in the 1960s, their relevance to cartographic applications has only recently become an explicit focus of research. Moreover, the potential of VR technology to visualize topographic databases has yet to be explored by cartographers.
\end{abstract}

In this experiment, we designed a VR application of a fictitious city derived from state 1:10,000 topographic data (Polish Database of Topographic Objects BDOT10k) to test user preferences for 2D or 3D urban geovisualizations. The app allows the user to switch between $2 \mathrm{D}$ and 3D representations of buildings in the simulation using a remote controller. This functionality enabled participants of the experiment to freely select 2D or 3D mode and for their preferences to be recorded and measured.

Our experiment involved two groups, one based in Poland and one in the UK, each comprising 30 participants (students enrolled on a Geography undergraduate course at each author's institution). Participants performed spatial ability tests to help ensure consistency in the sample and each group was divided into two sub-groups. Participants in the first subgroup were each given a navigation task that required their movement across the simulated city from point A to point B in the shortest possible time. Those in the second sub-group were given the freedom to explore the simulated city without being given a specific navigational task. We then interviewed participants in order to understand their own perception of their experiences in using the app.

The results indicate the preferences of the two groups and sub-groups of participants. In particular, we establish whether users preferred the 2D mode for the navigational task and the 3D mode for free exploration. The findings suggest how producers of topographic datasets might develop the functionality of their products using VR. 